\title{
Artificially structured boundary for confinement of effectively unmagnetized cryogenic antimatter plasmas
}

R. M. Hedlof, and C. A. Ordonez

Citation: AIP Conference Proceedings 1928, 020003 (2018);

View online: https://doi.org/10.1063/1.5021568

View Table of Contents: http://aip.scitation.org/toc/apc/1928/1

Published by the American Institute of Physics

Articles you may be interested in

Preface: Non-Neutral Plasma Physics X

AIP Conference Proceedings 1928, 010001 (2018); 10.1063/1.5021564

Recent experiments with lithium ion and electron plasmas in the BX-U linear trap

AIP Conference Proceedings 1928, 020005 (2018); 10.1063/1.5021570

Using chaos theory to analyze particle dynamics in asymmetry-induced transport

AIP Conference Proceedings 1928, 020001 (2018); 10.1063/1.5021566

Conference Photo: Non-Neutral Plasma Physics X

AIP Conference Proceedings 1928, 010002 (2018); 10.1063/1.5021565

Progress of the APEX experiment for creation of an electron-positron pair plasma

AIP Conference Proceedings 1928, 020004 (2018); 10.1063/1.5021569

Toward a compact levitated superconducting dipole for positron-electron plasma confinement

AIP Conference Proceedings 1928, 020015 (2018); 10.1063/1.5021580 


\title{
Artificially Structured Boundary for Confinement of Effectively Unmagnetized Cryogenic Antimatter Plasmas
}

\author{
R. M. Hedlof and C. A. Ordonez ${ }^{\mathrm{a})}$ \\ Department of Physics, University of North Texas, Denton, Texas 76203, USA \\ ${ }^{\text {a) }}$ Corresponding author: cao.unt.edu
}

\begin{abstract}
An artificially structured boundary (ASB) produces a short-range, static electromagnetic field that can reflect charged particles. In the work presented, an ASB is considered to consist of a spatially periodic arrangement of electrostatically plugged magnetic cusps. When used to create an enclosed volume, an ASB may confine a non-neutral plasma that is effectively free of applied electromagnetic fields, provided that the spatial period of the ASB-applied field is much smaller than any one dimension of the plasma. As envisioned, a non-neutral positron plasma could be confined by an ASB along its edge, and the space charge of the positron plasma would serve to confine an antiproton plasma. If the conditions of the two-species plasma are suitable, production of antihydrogen via three-body recombination for antimatter gravity studies may be possible. Particle-in-cell (PIC) simulations are used to study radial confinement of non-neutral and partially neutralized plasmas in a cylindrically symmetric ASB trap. For the timescales and plasma conditions considered in this work, plasma losses are found to be negligible.
\end{abstract}

\section{INTRODUCTION}

Non-neutral plasmas are routinely confined within cylindrically symmetric Penning traps. Penning traps employ a strong uniform magnetic field that is directed along the axis of symmetry of the configuration for radial confinement, and a sequence of electrodes to axially confine the plasma. For some applications, however, it may be desirable to employ a confinement scheme in which the bulk of the confinement volume is free of externally applied electromagnetic fields. One application that may benefit from such a confinement scheme is the production of antihydrogen for gravity experiments. As the temperature of the antiproton and positron plasmas is reduced, three-body recombination (TBR) becomes the dominant mechanism by which antihydrogen is produced. For a given density, the TBR rate is dependent on the temperature of the positron plasma and the strength of the magnetic field in which the TBR event occurs. Theoretical studies indicate that the TBR rate is reduced by approximately an order of magnitude when a strong magnetic field is present as opposed to the case when no magnetic field is present $[1,2]$.

A concept referred to as an artificially structured boundary (ASB) may lead to a method for producing antihydrogen in a region of space that is effectively free of externally applied electromagnetic fields. An ASB creates a static electromagnetic field that is short in range, and can be used to reflect charged particles. In the context of this work, an ASB can be considered to consist of a spatially periodic arrangement of electrostatically plugged magnetic cusps. The dimensions of a plasma trap that utilizes an ASB are chosen such that the spatial period of the applied electromagnetic field is much smaller than any one dimension of the confinement volume. Such a configuration creates a confinement volume that is largely free of externally applied electromagnetic fields.

Here, radial plasma confinement within a cylindrically symmetric ASB trap is investigated via computer simulation. A cross sectional view of the ASB configuration considered in this work is shown in Fig. 1. The magnetic field of the ASB is produced by a sequence of 30 circular current carrying coils of radius $R_{b}=5.0 \mathrm{~cm}$ that are all coaxial with the $z$-axis. The coils are spaced equidistant from one another at $0.5 \mathrm{~cm}$ intervals along the $z$-axis. Each of the coils carries a total (single-loop) current of $I=750 \mathrm{~A}$, and adjacent coils carry current in opposite directions. This arrangement of electromagnets creates a magnetic field that is spatially periodic in the $z$ direction with a spatial period of $S=1.0 \mathrm{~cm}$, and has a sequence of magnetic ring cusps located at each of the mid-planes between adjacent coils. To confine particles that would otherwise be lost through the magnetic ring cusps, a sequence of plugging electrodes (dark gray) are placed in the center of each ring cusp, and are biased to $V_{e}=75 \mathrm{~V}$. The electrodes have an inner radius

Non-Neutral Plasma Physics X

AIP Conf. Proc. 1928, 020003-1-020003-6; https://doi.org/10.1063/1.5021568 Published by AIP Publishing. 978-0-7354-1620-8/\$30.00

020003-1 
of $R_{T}=4.95 \mathrm{~cm}$, and are $d=1.5 \mathrm{~mm}$ thick in the $z$-dimension. The plugging electrodes are mounted flush with another sequence of cylindrical electrodes (light gray) that are held at zero potential, and are $l=3.5 \mathrm{~mm}$ thick in the $z$-dimension. The total length of the trap is $14.5 \mathrm{~cm}$.
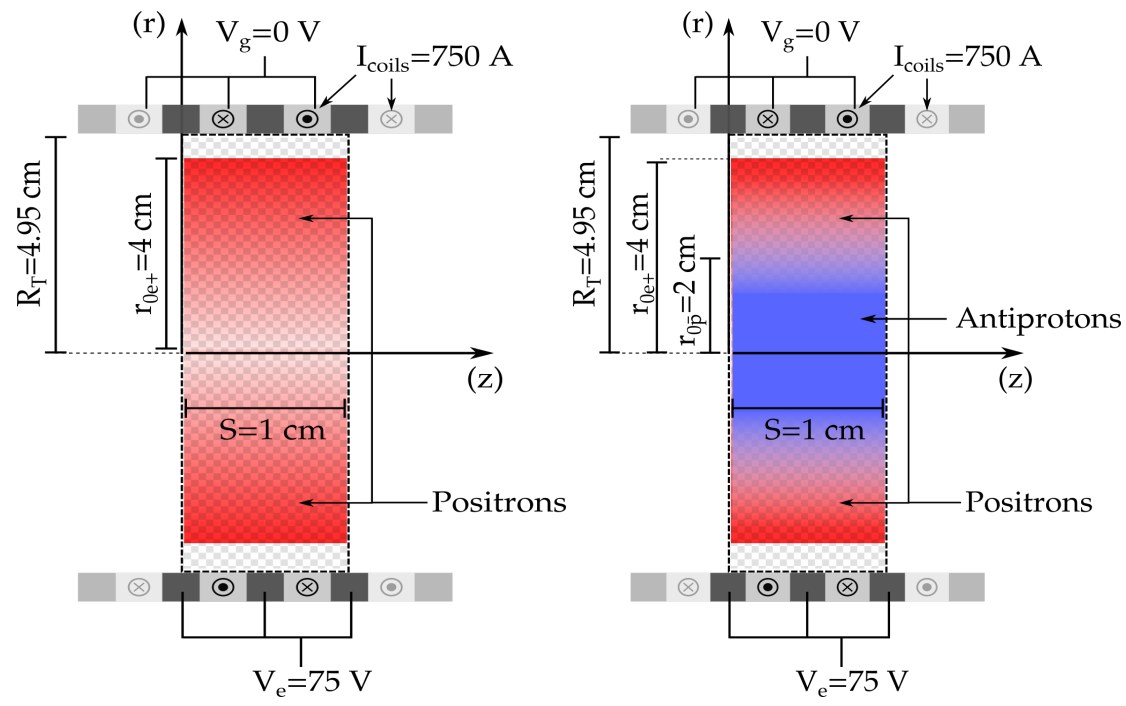

FIGURE 1. A conceptual illustration of the ASB used for confining (left) a non-neutral positron plasma, and (right) a partially neutralized positron-antiproton plasma.

\section{SIMULATION MODEL}

Each of the circular current carrying coils in Fig. 1 is approximated as an infinitesimally thin circular wire of radius $R_{b}$. The components of the magnetic field $\mathbf{B}_{s}$ produced by an infinitesimally thin circular wire carrying current in the counter-clockwise sense, that lies in the $z=0$ plane, and that is centered about the origin of a cylindrical coordinate system are given, for example, in Ref. [3]. The total magnetic field of the trap is given by the superposition of the fields due to each of the individual loops

$$
\mathbf{B}(r, z)=\sum_{i=1}^{N_{c}}(-1)^{i+1} \mathbf{B}_{s}\left(r, z-z_{i}\right),
$$

where $N_{c}$ is the number of circular coils comprising the trap, and $z_{i}$ is the $z$ coordinate of the $i^{\text {th }}$ coil. The expression $z_{i}=S\left[2(i-1)+3-N_{c}\right] / 4$ is used in Eq. (1) so that the center of the simulation volume is co-planar with the cusp in the center of the trap. Figure 2 (left) shows the magnitude of the magnetic field in the $z=0.5 \mathrm{~cm}$ plane as a function of radius. In the $z=0.5 \mathrm{~cm}$ plane, the magnitude of the magnetic field drops by $98.9 \%$ from a value of $B=0.095 \mathrm{~T}$ in the cusp at $r=R_{T}$ to $B=10^{-3} \mathrm{~T}$ at $r=4 \mathrm{~cm}$. The magnitude of the magnetic field has a maximum value along the $z$-axis of $B=2.2 \times 10^{-4} \mathrm{~T}$.

The particle-in-cell (PIC) code Warp [4] has been used for all simulations in this work. The simulations are conducted with a two-dimensional electrostatic version of Warp, whereby the electrostatic potential is calculated selfconsistently in cylindrical coordinates and the self-induced magnetic field of the plasma is neglected. The electrostatic potential is calculated on a square mesh in cylindrical coordinates, where the grid size $\Delta r=\Delta z=\Delta s$ is chosen according to $\Delta s=\min \left(\lambda_{D \sigma} / 4, S / N_{S}\right)$, where min is a function returning the smallest value among its arguments, $N_{S}$ is the number of grid points per spatial period used in the simulation, and $\lambda_{D \sigma}=\sqrt{\epsilon_{0} k_{B} T_{\sigma 0} /\left(n_{\sigma 0} e^{2}\right)}$ is the smallest species Debye length in the simulation. Here, $T_{\sigma 0}$ and $n_{\sigma 0}$ are the initial temperature and density of the species $\sigma$. The grid size is chosen such that there are at least four grid points per Debye length in each direction, and at least $N_{S}=90$ grid points per spatial period of the magnetic field. Dirichlet boundary conditions are imposed on the electrostatic 

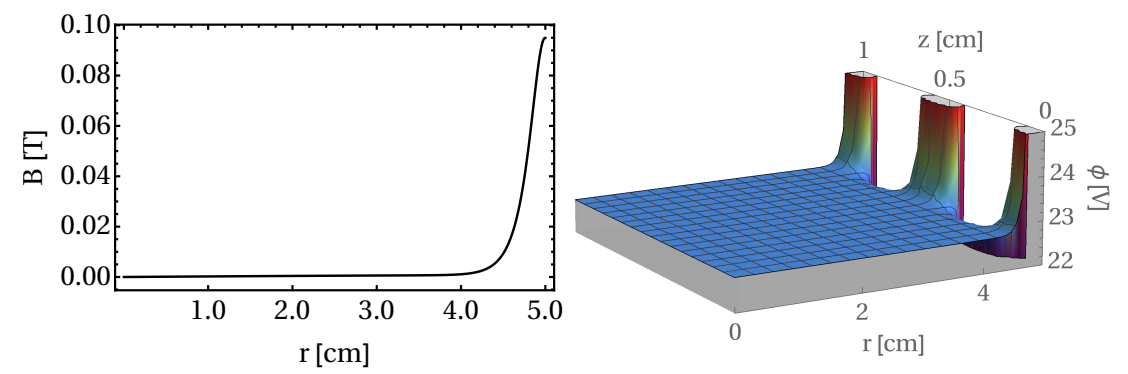

FIGURE 2. (left) Magnitude of the magnetic field in the central cusp and (right) electrostatic potential of the empty trap.

potential at $r=R_{T}$ as shown in Fig. 1, and Neumann boundary conditions are imposed on the electrostatic potential in the $z=0.0 \mathrm{~cm}$ and $z=1.0 \mathrm{~cm}$ planes such that $\partial_{z} \phi(r, 0.0 \mathrm{~cm})=\partial_{z} \phi(r, 1.0 \mathrm{~cm})=0.0 \mathrm{~V} / \mathrm{m}$. Figure 2 (right) shows the electrostatic potential of the empty trap.

When initializing the simulations, plasma particles are loaded into the trap within a cylindrically symmetric plasma column of radius $r_{0 \sigma}$ according to a uniform radial density profile over the course of $2 \mu$ s unless otherwise stated. The total number of particles of each species that are loaded into the simulation volume is chosen such that each plasma would have a density of $n_{0 \sigma}=10^{6} \mathrm{~cm}^{-3}$ had the plasma been initialized instantaneously within the corresponding injection column at $t=0 \mathrm{~s}$. Each simulated particle, referred to hereafter as a macroparticle, in the PIC method represents a larger number of real particles. The number of real particles each simulated particle represents, is referred to as the weight $w$. A weight of $w=10$ for each macroparticle is used in all simulations. For all simulations presented in this work, the initial Cartesian velocity components $v_{0 x}, v_{0 y}$, and $v_{0 z}$ for all particles are sampled from a three-dimensional Maxwellian velocity distribution associated with a temperature of $T_{\sigma}=40 \mathrm{~K}$. Each particle in the simulations is allowed to move in three-dimensions under the combined effects of the ASB applied electromagnetic field and the self-consistently calculated electric field of the plasma. Reflecting boundary conditions are imposed for the plasma particles on the $z=0$ and $z=1.0 \mathrm{~cm}$ planes, and if plasma particles reach the surface of the electrodes at $r=R_{T}$ they are removed from the simulation.

While the bulk of each plasma is unmagnetized, some particles that are near the trap walls become magnetized. As such, the drift-Lorentz pusher [4] is used, which allows the use of a timestep equal to, or larger than, the cyclotron period when advancing particles. The timestep $\Delta t$ at $t=0$ is chosen according to $\Delta t=\min \left(\Delta t_{p 0}, \Delta t_{c}, \Delta t_{C F L, 0}\right)$, where $\Delta t_{p 0}=0.1 / \omega_{p 0}$ is the timestep required to resolve the positron plasma period, $\omega_{p 0}=\sqrt{n_{0 e^{+}} e^{2} / \epsilon_{0} m_{e^{+}}}$is the positron plasma frequency had the positrons been initialized instantaneously at $t=0$ with a density $n_{0 e^{+}}=10^{6} \mathrm{~cm}^{-3}$, $\Delta t_{c}=2 \pi m_{e^{+}} /\left(e B\left(\mathbf{r}_{e d g e}\right)\right)$ is the cyclotron period of a positron if it were located near the edge of the trap at $\mathbf{r}_{e d g e}=$ $\left(0.99 R_{T}, 0.25 S\right), \Delta t_{C F L, 0}=\Delta s / v_{C F L, 0}$, and $v_{C F L, 0}=500,000 \mathrm{~m} / \mathrm{s}$ is a velocity chosen sufficiently large that a very small portion of the plasma population initially violates the Courant-Friedrichs-Lewy (CFL) condition. To ensure that the CFL condition is met as the plasma evolves, the timestep is continuously updated every 100 timesteps according to $\Delta t=\min \left(\Delta t_{p 0}, \Delta t_{c}, \Delta s / v_{\max }\right)$, where $v_{\max }$ is the speed of the fastest particle in the simulation volume.

Two plasma confinement simulations are presented in this work, and will hereafter be referred to as Case I, and Case II, respectively. In Case I, confinement of a non-neutral positron plasma is modelled. Positron $\left(e^{+}\right)$macroparticles are injected over the course of the first $2 \mu$ s of the simulation into a cylindrically symmetric plasma column of radius $r_{0 e^{+}}=4.0 \mathrm{~cm}$. The plasma is then allowed to evolve for another $18 \mu \mathrm{s}$.

In Case II, the possibility of using the space-charge of the $e^{+}$plasma to confine antiprotons $(\bar{p})$ is investigated. The evolution of the $e^{+}-\bar{p}$ system is very slow on the time-scales necessary to resolve the $e^{+}$dynamics. As such, an electron-positron $\left(e^{-}-e^{+}\right)$plasma is first simulated, and after the $e^{-}-e^{+}$system evolves for some time, the simulation is stopped, and the electron phase-space distribution is used to approximate the initial state of the $\bar{p}$ plasma. This is accomplished as follows: First, the $e^{-}-e^{+}$plasmas are injected simultaneously into two plasma injection columns of radii $r_{0 e^{-}}=2.0 \mathrm{~cm}$ and $r_{0 e^{+}}=4.0 \mathrm{~cm}$, respectively. The system is allowed to evolve for $18 \mu \mathrm{s}$, the simulation is stopped, and the phase-space of each species is recorded. Each macroparticle in the $e^{+}$plasma is then re-initialized exactly as it was in the previous run, and $\bar{p}$ macroparticles are initialized with the same spatial coordinates and kinetic energy as the electrons from the previous run, with the velocity of the $i^{\text {th }} \bar{p}$ chosen according to $\mathbf{v}_{i, \bar{p}}=\sqrt{m_{e^{+}} / m_{\bar{p}}} \mathbf{v}_{i, e^{-}}$. The $e^{+}-\bar{p}$ system is then allowed to evolve for another $10.2 \mu \mathrm{s}$. To speed up the final stage of the simulation in Case II, 
a maximum speed $v_{C F L, \max }=1.5 \times 10^{6} \mathrm{~m} / \mathrm{s}$ for all particles in the system is implemented. If the speed of any particle surpasses $v_{C F L \text {, max }}$, it is removed from the simulation.

\section{RESULTS}

\subsection{Case I: Non-neutral Positron Plasma Confinement}
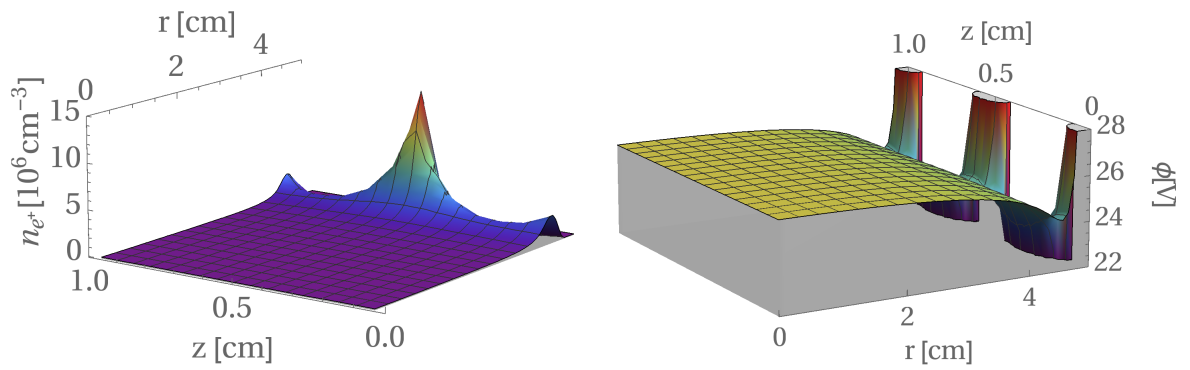

FIGURE 3. (left) Positron density and (right) electrostatic potential within the simulation volume at $t=20 \mu \mathrm{s}$.

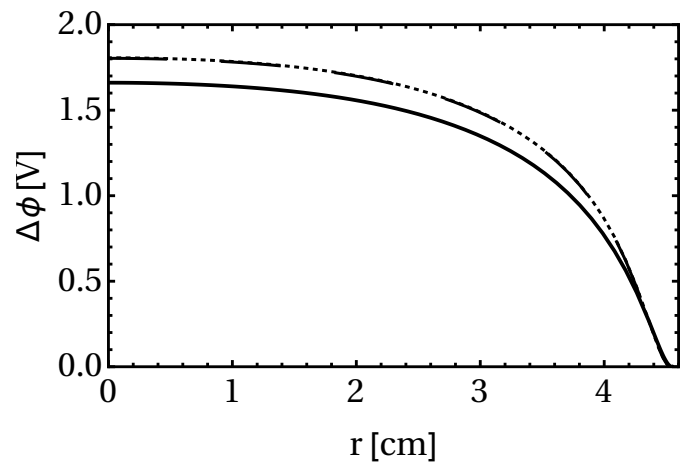

FIGURE 4. Depth of the electrostatic potential well formed by the space-charge of a non-neutral positron plasma at $t=20 \mu \mathrm{s}$ in the $z=0.0 \mathrm{~cm}$ (dotted), $z=0.5 \mathrm{~cm}$ (solid), and $z=1.0 \mathrm{~cm}$ (dashed) planes.

In Case I, a total of 5,026,626 positron macroparticles are injected into the cylindrically symmetric injection column as shown in Fig. 1 (left). No particles were lost over the course of the $18 \mu$ s confinement time after particle injection ceased at $2 \mu \mathrm{s}$. Figure 3 (left) shows the density of the positron plasma $n_{e^{+}}(r, z)$ in the simulation volume at $t=20 \mu \mathrm{s}$. The positrons tend to accumulate in the central cusp, where there is a peak in the density profile of $n_{e^{+}}(4.5 \mathrm{~cm}, 0.5 \mathrm{~cm})=1.43 \times 10^{7} \mathrm{~cm}^{-3}$. The density profile also has two local maxima in the cusps along the edges of the simulation volume with densities of $n_{e^{+}}(4.42 \mathrm{~cm}, 0.0 \mathrm{~cm})=2.94 \times 10^{6} \mathrm{~cm}^{-3}$, and $n_{e^{+}}(4.35 \mathrm{~cm}, 1.0 \mathrm{~cm})=2.84 \times$ $10^{6} \mathrm{~cm}^{-3}$. The density near the radial center of the trap is much lower, with an average value of $\left\langle n_{e^{+}}\right\rangle=4.83 \times 10^{4} \mathrm{~cm}^{-3}$ along the $z$-axis.

Figure 3 (right) shows the electrostatic potential $\phi(r, z)$ within the simulation volume at $t=20 \mu$ s. The electrostatic potential has local minima in each of the cusp planes located at $z=0.0 \mathrm{~cm}, z=0.5 \mathrm{~cm}$, and $z=1.0 \mathrm{~cm}$. These minima define the depth of the electrostatic potential well that can be used to confine an oppositely signed species in the radial direction, and have values of $\phi(4.54 \mathrm{~cm}, 0.0 \mathrm{~cm})=\phi(4.54 \mathrm{~cm}, 1.0 \mathrm{~cm})=24.2 \mathrm{~V}$, and $\phi(4.55 \mathrm{~cm}, 0.5 \mathrm{~cm})=24.4 \mathrm{~V}$. The electrostatic potential varies by less than $1.1 \times 10^{-3} \%$ along the axis of symmetry of the configuration, and has a value of $\phi(0.0 \mathrm{~cm}, 0.5 \mathrm{~cm})=26.0 \mathrm{~V}$ in the plane of the central cusp. The 
difference between trap center, and the minima in the $z=0.0 \mathrm{~cm}$ (dotted), $z=0.5 \mathrm{~cm}$ (solid), and $z=1.0 \mathrm{~cm}$ (dashed) planes are shown in Fig. 4. The radial depth of the space-charge well in the $z=0.0 \mathrm{~cm}, z=0.5 \mathrm{~cm}$, and $z=1.0 \mathrm{~cm}$ planes are, respectively, $\Delta \phi_{0.0}=1.80 \mathrm{~V}, \Delta \phi_{0.5}=1.66 \mathrm{~V}$, and $\Delta \phi_{1.0}=1.81 \mathrm{~V}$.

\subsection{Case II: Space-charge Based Antiproton Confinement}
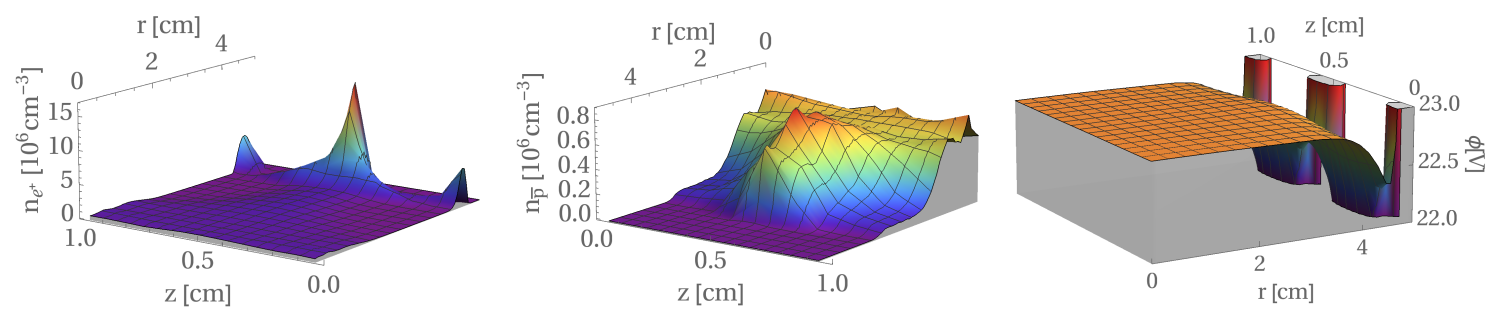

FIGURE 5. Positron density (left), antiproton density (center), and electrostatic potential (right) within the trap volume at $10.2 \mu \mathrm{s}$ for Case II.

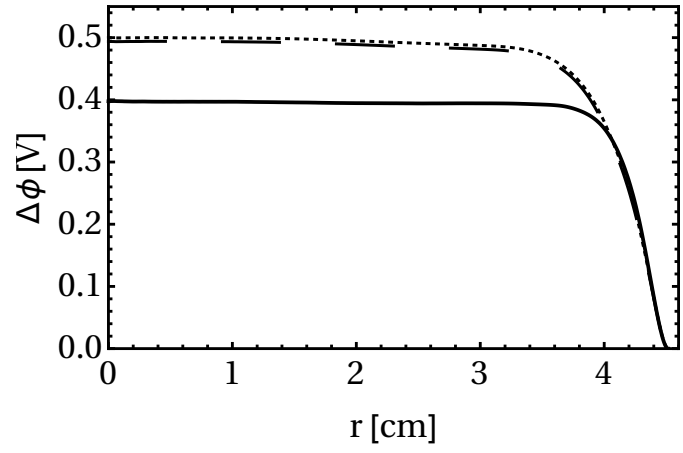

FIGURE 6. Depth of the electrostatic potential well for Case II at $t=10.2 \mu \mathrm{s}$ in the $z=0.0 \mathrm{~cm}$ (dotted), $z=0.5 \mathrm{~cm}$ (solid), and $z=1.0 \mathrm{~cm}$ (dashed) planes.

In Case II, 5, 026,610 $e^{+}$and 1,256,653 $\bar{p}$ are re-injected into the simulation volume after the initial $e^{-}-e^{+}$ simulation is completed. Figure 5 shows the density profiles of the positron (left) and antiproton (center) plasmas at $t=10.2 \mu \mathrm{s}$. The positron plasma density $n_{e^{+}}(r, z)$ has maxima in the $z=0.0, z=0.5$, and $z=1.0$ planes with values of $n_{e^{+}}(4.41 \mathrm{~cm}, 0.0 \mathrm{~cm})=5.74 \times 10^{6} \mathrm{~cm}^{-3}, n_{e^{+}}(4.56 \mathrm{~cm}, 0.5 \mathrm{~cm})=1.60 \times 10^{7} \mathrm{~cm}^{-3}$, and $n_{e^{+}}(4.41 \mathrm{~cm}, 1.0 \mathrm{~cm})=$ $5.55 \times 10^{6} \mathrm{~cm}^{-3}$, respectively. Near the axis of symmetry of the trap, the $e^{+}$and $\bar{p}$ plasmas have approximately the same density with $\left\langle n_{e^{+}}\right\rangle=5.64 \times 10^{5} \mathrm{~cm}^{-3}$ and $\left\langle n_{\bar{p}}\right\rangle=5.41 \times 10^{5} \mathrm{~cm}^{-3}$. The maximum $r$-coordinate of all $\bar{p}$ in the simulation is $r_{\bar{p}, \max }=3.86 \mathrm{~cm}$. Throughout the simulation 246 positrons were removed from the simulation volume because their speed was greater than $v_{C F L \text {,max }}$. No antiprotons were lost.

The electrostatic potential $\phi(r, z)$ for Case II is shown in Fig. 5 (right) at $10.2 \mu \mathrm{s}$. As in the previous section, the electrostatic potential has local minima in the $z=0.0, z=0.5$, and $z=1.0$ planes with values $\phi(4.52 \mathrm{~cm}, 0.0 \mathrm{~cm})=$ $22.4 \mathrm{~V}, \phi(4.52 \mathrm{~cm}, 0.5 \mathrm{~cm})=22.5 \mathrm{~V}$, and $\phi(4.52 \mathrm{~cm}, 1.0 \mathrm{~cm})=22.3 \mathrm{~V}$, respectively. The potential difference between trap center, and the minima located at $z=0.0 \mathrm{~cm}$ (dashed), $z=0.5 \mathrm{~cm}$ (solid), and $z=1.0 \mathrm{~cm}$ (dotted) are shown in Fig. 6. The depth of the electrostatic well in each of these planes is $\Delta \phi_{0.0}=0.49 \mathrm{~V}, \Delta \phi_{0.5}=0.40 \mathrm{~V}$, and $\Delta \phi_{1.0}=0.50 \mathrm{~V}$, respectively. 


\section{DISCUSSION AND CONCLUDING REMARKS}

An artificially structured boundary (ASB) has been presented that may lead to a method for confining non-neutral and partially neutralized plasmas that are effectively unmagnetized. The system has been modeled with the PIC code Warp. The results presented in Section 3.1 and 3.2 indicate that it may be possible to confine non-neutral and partially neutralized plasmas with negligible losses in the radial direction by employing an ASB trap. An electrostatic potential well is formed by the space-charge of the positron plasma, that may be used to radially confine an oppositely signed species. The smallest value for $\Delta \phi$ defines the depth of the space-charge potential. For both Case I (non-neutral) and Case II (partially neutralized) plasmas, the depth of the space-charge well was smallest along the $z=0.5 \mathrm{~cm}$ plane, with values of $\Delta \phi=1.66 \mathrm{~V}$ and $\Delta \phi=0.40 \mathrm{~V}$, respectively. It is expected that radial losses of the space-charge confined antiprotons would be negligible so long as $e \Delta \phi \gg k_{B} T_{\bar{p}}$. While the simulations indicate that space-charge based confinement of antiprotons may be possible, further work is necessary to determine whether a space-charge well will exist in the axial direction, and what the depth of the axial well would be. It should be noted that, if the ASB trap is constructed in a toroidal shape it would not be necessary to confine the plasmas along the axial direction.

\section{ACKNOWLEDGMENTS}

The authors would like to thank Ryan Phillips (UNT) and Dr. David Grote (LLNL) for helpful discussions regarding the Warp simulations. Computational resources were provided by UNT's High Performance Computing Services. This material is based upon work supported by the National Science Foundation under Grant No. PHY-1500427 and by the Department of Energy under Grant No. DE-FG02-06ER54883.

\section{REFERENCES}

[1] M. E. Glinsky and T. M. O’Neil, Physics of Fluids B 3, 1279-1293 (1991).

[2] F. Robicheaux, Phys. Rev. A 73, p. 033401 (2006).

[3] C. A. Ordonez, D. D. Dolliver, Y. Chang, and J. R. Correa, Physics of Plasmas 9, 3289-3302 (2002).

[4] A. Friedman, R. H. Cohen, D. P. Grote, S. M. Lund, W. M. Sharp, J. L. Vay, I. Haber, and R. A. Kishek, IEEE Transactions on Plasma Science 42, 1321-1334 (2014). 